\title{
Tumors in Epilepsy
}

\author{
Mark Nowell, MBBS, MA, MRCS ${ }^{1,2,3}$ Anna Miserocchi, MD ${ }^{1,2,3}$ Andrew W. McEvoy, FRCS ${ }^{1,2,3}$
}

${ }^{1}$ Department of Clinical and Experimental Epilepsy, UCL Institute of Neurology, London, United Kingdom

2 Department of Neurosurgery, National Hospital for Neurology and

Address for correspondence Mark Nowell, MBBS, MA, MRCS, Box 29, National Hospital for Neurology and Neurosurgery, Queen Square, Neurosurgery, London, United Kingdom

${ }^{3}$ MRI Unit, Epilepsy Society, Chalfont St Peter, United Kingdom

Semin Neurol 2015;35:209-217.

\begin{abstract}
Keywords

- epilepsy

- tumors

- surgery

Primary brain tumors are common causes of focal epilepsy, accounting for $5 \%$ of newonset seizures in adults and over $10 \%$ of lesional focal epilepsies. These epilepsies are often refractory to medical treatment, and high rates of seizure freedom can be achieved with gross total resections. However, the management strategy is not straightforward, and should be decided on a case-by-case basis in a multidisciplinary team, considering the natural history of the tumor, the likelihood of seizure freedom following surgical resection, the risks of surgery, and the impact of seizures on quality of life. In this review, the authors summarize the crucial factors that help to decide how to manage this challenging patient group.
\end{abstract}

\section{Tumor Classification}

All types of primary and secondary brain tumor have the potential to cause seizures. However, for the purposes of this review we shall discuss only the most common epileptogenic tumors. Primary tumors can be divided into the rarer, benignacting tumors, termed long-term epilepsy-associated tumors (LEATs), ${ }^{1}$ and the more common low-grade and high-grade astrocytic tumors encountered in neuro-oncological practice.

Long-term epilepsy-associated tumors typically present in young patients with seizures. Imaging shows cortically based, well-circumscribed lesions, often in the temporal lobe, with no evidence of diffuse infiltration ( $\mathbf{- F i g . 1}$ ). These tumors can be further subdivided into those of mixed glioneuronal origin and those of glial origin. Glioneuronal tumors have the highest seizure rate, most likely due to hyperexcitable regions of dysplastic neurons.

Low-grade gliomas are World Health Organization (WHO) grade II tumors, and comprise diffuse astrocytoma (seizures seen in $50-81 \%$ ), oligodendroglioma (seizures in $46-78 \%)^{2}$ and mixed oligoastrocytoma. These slow-growing tumors infiltrate into surrounding cortex and along white matter tracts, and ultimately undergo malignant transformation into grades III and IV. The median survival for a diffuse astrocytoma is 8.2 years ( $\mathbf{- F i g . ~} \mathbf{2 A}$ ). ${ }^{3}$ - Table 1 shows the epidemiological and pathological characteristics of the LEATs and low-grade gliomas. ${ }^{1,2,4}$

Common malignant tumors comprise the WHO grade III and IV glial tumors, and include anaplastic astrocytoma, anaplastic oligodendroglioma, anaplastic oligoastrocytoma, and glioblastoma multiforme (GBM). These tumors are considered to be less epileptogenic than the LEATs and low-grade gliomas, with reported seizure rates of 22 to $62 \%,{ }^{2}$ although this may reflect the shorter life span of the patients (median survival for patients with GBM with best treatment is 14.6 months. $)^{5}$ Like low-grade gliomas, these tumors also infiltrate along white matter tracts, but they are far more aggressive, causing localized tissue destruction, ischemia, and necrosis (-Fig. 2B).

\section{Pathophysiology}

The pathophysiology of tumor epileptogenesis is multifactorial, and certain mechanisms are probably more prevalent in certain tumor types. Long-term epilepsy-associated tumors of glioneural origin are highly epileptogenic. This is thought to be a predominantly direct effect, through hyperexcitable regions of dysplastic neurones developing inside the tumor itself. However, sometimes there is an additional peritumoral component. Upregulation of gap junctions locally may lead to
Issue Theme Etiology of Epilepsy; Guest Editors: Philip Smith, MD, FRCP,

FAcadMEd, and Rhys Thomas, BSc, MRCP, MSc, PhD
Copyright $\odot 2015$ by Thieme Medical Publishers, Inc., 333 Seventh Avenue, New York, NY 10001, USA. Tel: +1(212) 584-4662.
DOI http://dx.doi.org/ $10.1055 / \mathrm{s}-0035-1552628$. ISSN 0271-8235. 

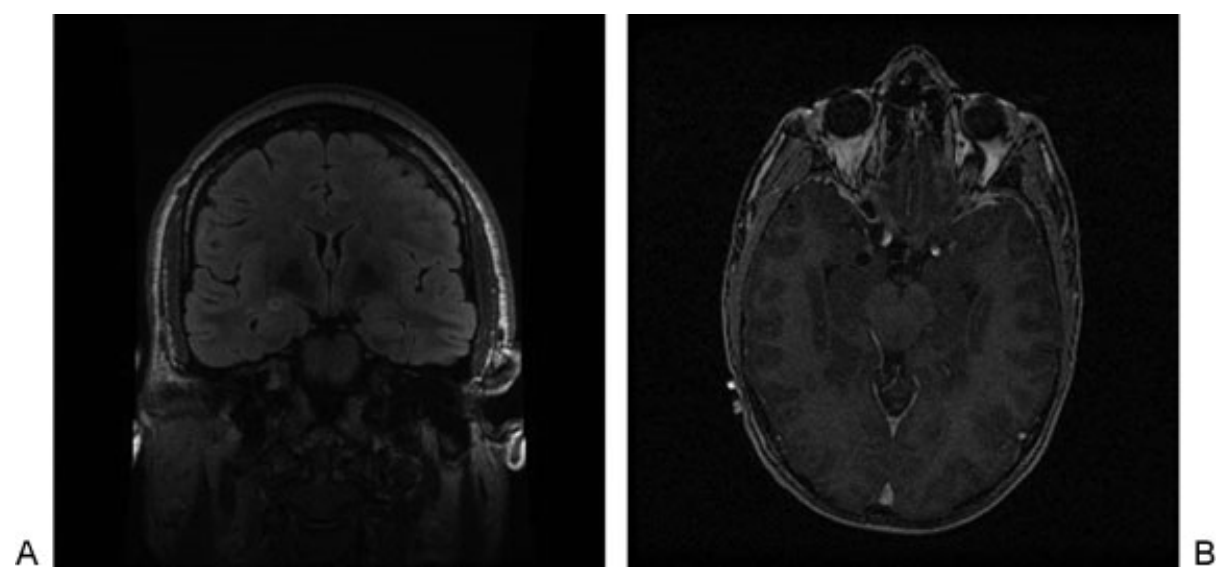

Fig. 1 (A) Coronal T2 fluid-attenuated inversion-recovery view of right-sided medial temporal dysembryoplastic neuroepithelial tumor. (B) Axial T1-weighted magnetic resonance image with gadolinium contrast of right-sided mesial temporal ganglioglioma. Note the small degree of gadolinium enhancement.

peritumoral neural networks and seizure propagation outside of the tumor. ${ }^{2}$ Some cases have associated focal cortical dysplasia $(F C D)$, recently classified by the International League Against Epilepsy (ILAE) as FCD type IIIb. ${ }^{6}$

Gliomas have no intrinsic neural component, and so epileptogenesis must result from tumor infiltration into surrounding cortex, with an indirect effect on the surrounding neural networks. There is good evidence from magnetoencephalography, surface electroencephalography, and stereo-electroencephalography that epileptiform activities in gliomas arise from the peritumoral cortex. ${ }^{7}$ There are many candidates for potential mediators of epileptogenesis, and they can be broadly grouped into an epileptocentric and tumorcentric framework. ${ }^{7}$

\section{Epileptocentric Causes}

The epileptocentric hypothesis is that seizures arise from a change in the local excitability of the peritumoral milieu. This is primarily due to a state of disequilibrium, caused by the upregulation of excitatory glutaminergic transmission, coupled with decreased GABA transmission. GABAergic transmission may play a more active role: Disturbances in chloride homeostasis may lead to a pathological switch from an inhibitory hyperpolarizing effect of chloride influx to an excitatory depolarizing effect of chloride efflux. Other factors may also exacerbate local excitability, including $\mathrm{pH}$ shifts, increased gap junction-mediated connectivity and bloodbrain barrier disruption. ${ }^{2}$ The epileptocentric framework for tumor epileptogenesis is most applicable to low-grade
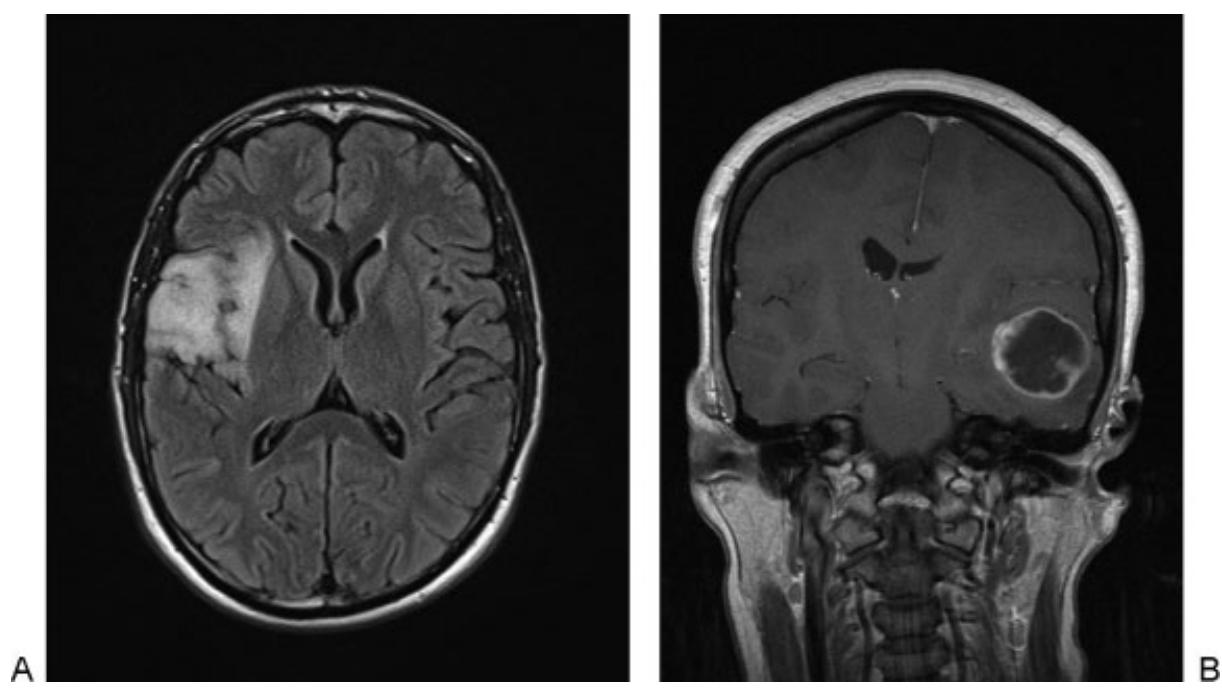

Fig. 2 (A) Axial T2 fluid-attenuated inversion-recovery view of right-sided insular low-grade glioma with extension into the temporal pole. (B) Coronal T1-weighted magnetic resonance image with gadolinium contrast of left-sided temporal high-grade glioma. Note the rim-enhancing lesion causing local mass effect and subfalcine herniation. 
Table 1 Table to show the epidemiology and pathophysiology of the long-term epilepsy-associated tumors

\begin{tabular}{|l|l|l|l|l|l|}
\hline Type & Origin & WHO Grade & Frequency & Most common location & $\begin{array}{l}\text { Malignant } \\
\text { transformation }\end{array}$ \\
\hline $\begin{array}{l}\text { Dysembryoplastic } \\
\text { neuroepithelial tumor }\end{array}$ & Glioneural & I & $15 \%$ & Temporal & Very rare ${ }^{28}$ \\
\hline Ganglioglioma & Glioneural & I & $40 \%$ & Temporal & Rare \\
\hline Gangliocytoma & Glioneural & I & Rare & Temporal & \\
\hline Angiocentric glioma & Glioneural & I & $<3 \%$ & Temporal & Unknown \\
\hline Pilocytic astrocytoma & Glial & I & $16 \%$ & Around third ventricle & Rare \\
\hline Diffuse astrocytoma & Glial & II & $16 \%$ & Frontal, temporal & Certain \\
\hline Oligodendroglioma & Glial & II & $11 \%$ & Frontal, temporal & Certain \\
\hline Pleomorphic xanthoastrocytoma & Glial & II & $2 \%$ & Temporal & Rare \\
\hline
\end{tabular}

gliomas, where there is slow growth, with gliosis and chronic inflammation in the peritumoral cortex.

\section{Tumorocentric Causes}

The tumorocentric hypothesis is that epilepsy arises from the direct mechanical effects of the space-occupying lesion. Local mass effect and edema lead to regional intracranial hypertension and corresponding cerebral hypoperfusion. There is localized tissue destruction, ischemia, and necrosis, and a simultaneous neoangiogenesis that may induce microhemorrhages and further inflammatory changes. Structural reorganization and functional deafferentation are well described, with the recruitment of nonmalignant cells, such as astrocytes and microglia, further facilitating intercellular communication and seizure propagation. The tumorocentric framework for tumor epileptogenesis is most applicable to high-grade gliomas, which grow rapidly and cause significant local disruption.

\section{Prognostic Factors}

The natural course of epilepsy associated with brain tumors differs from other epilepsies; surgical resection offers a potentially curative treatment. There are several clear prognostic factors for seizure freedom following surgery. First, tumor type is important, with good rates of seizure freedom seen in LEATs and WHO grade I gliomas. Surgery on dysembryoplastic neuroepithelial tumors (DNETs) gives an average seizure freedom of $88 \%$ at 5 years (range $58-90 \%$ ); surgery on gangliogliomas gives seizure freedom in $79 \%$ at 5 years (range 45-100\%). ${ }^{8}$

Extended reviews of glioneural tumors indicate that the most important predictor of seizure freedom in this group is total removal of the tumor. ${ }^{9}$ Less important prognostic factors include reasonable preoperative seizure control, absence of secondary generalized seizures, and a short history of epilepsy ( $<1$ year). ${ }^{9}$ Patient age and tumor location do not appear to be important. There is no evidence that intraoperative electrocorticography (ECoG) makes any difference. ${ }^{10}$
Similarly, extended reviews of surgery for low-grade gliomas also indicate that gross total resections are the most important prognostic factor in achieving seizure freedom. ${ }^{10}$ In this context, gross total resection refers to the removal of the radiological abnormality because the pathology is infiltrative and diffuse. As is the case with LEATs, other predictors of seizure freedom include good preoperative control, absence of generalization, and a short duration of epilepsy. With both LEATs and low-grade gliomas in the temporal lobe, there is now reasonable evidence that adding an amygdalohippocampectomy to a gross total resection further improves seizure outcome ( $87 \%$ vs. $79 \%)^{10}$

The prognosis for seizure freedom in patients with highgrade glioma is less good because the pathology is destructive and widespread in the brain. The median survival of this group is poor; therefore, there are no data to document longterm seizure outcome following surgery.

\section{Importance of Holistic Care}

The diagnosis of tumor-related epilepsy is often challenging for patients because they carry with them the stigma of both epilepsy and tumor pathologies. Epilepsy diminishes quality of life for several reasons, including the impact on driving eligibility, employment and relationships, difficulty with independence, anxiety regarding seizures, and reliance upon antiepileptic drugs and their associated side effects. The diagnosis of a brain tumor brings additional concerns around the risk of malignant transformation and long-term survival. These patients often have neuropsychological issues due in part to the epilepsy and also to the use of antiepileptic drugs. Furthermore, changes in seizure pattern can trigger concerns in patients regarding tumor transformation or recurrence. Many would benefit from formal neuropsychological or neuropsychiatric assessment, and this factor should always be considered when asking patients to weigh up complex issues around their management. All patients with a diagnosis of tumor-related epilepsy should be discussed in a multidisciplinary team environment, with input from 
neurologists, neurophysiologists, neurosurgeons, neuroradiologists, and neuropyschologists. The remainder of this review focuses on management of tumor-related epilepsy, covering treatment aims, histological diagnosis, medical treatment, and surgical treatment.

\section{Treatment Aims}

The possible treatment aims in the management of tumorrelated epilepsy are summarized below:

1. Establish the histological diagnosis

2. Improve seizure control/achieve seizure freedom

3. Improve survival

4. Improve quality of life

The final aim, that is the improvement of quality of life, considers patients' posttreatment lives, and takes into account any newly acquired neurologic or cognitive deficits that may result from surgical treatment.

There is substantial overlap between these aims; for example, achieving seizure freedom will likely lead to an improvement of quality of life, and can also be viewed as improving survival, with a corresponding decrease in the risk of sudden unexpected death in epilepsy (SUDEP). Similarly, there is increasing evidence that total resection of low-grade gliomas can both improve seizure control and also prolong survival. $^{11}$

The priorities of treatment will clearly vary from case to case, and will be informed by patients' own ideas and concerns, as well as tumor type, pathophysiology, and likely prognosis. As a rule, in patients with a low-grade glioma, oncological clearance takes precedence, while for patients with LEATs it is the determination and removal of the epileptogenic zone. Functional preservation is paramount in both groups (-Fig. $\mathbf{3}$ ).

\section{Importance of Histological Diagnosis}

As previously mentioned, the histology and grade of the tumor is vital to determine natural history and select the appropriate management strategy. Structural magnetic resonance imaging (MRI) is probably sufficient to diagnose a LEAT, but is associated with up to a $50 \%$ false-positive rate in predicting the WHO grade in astrocytomas. ${ }^{12}$ To reduce the potential for misdiagnosis, there is therefore a clear indication to achieve a histological diagnosis in patients with epilepsy and structural imaging consistent with a glial tumor.

Aside from the risk of misdiagnosis, there is increasing interest in taking histological samples for the purpose of molecular and genetic profiling. DNA methylation of the $\mathrm{O}-$ methylguanine-DNA methyltransferase (MGMT) gene is well known as an independent predictor of improved survival in patients with grade IV glioma who are receiving temozolomide. ${ }^{5}$ Similarly, the $1 \mathrm{p} 19 \mathrm{q}$ deletion is associated with a more favorable survival in patients with anaplastic oligoastrocytoma. ${ }^{13}$ There is great value in identifying molecular markers in low-grade gliomas, to help determine likely growth rate and response to treatment, with much interest in the isocitrate dehydrogenase (IDH) mutational status. ${ }^{14}$ In the future, the genetic profile will be added to the tumor histology and grade to help to decide prognostic and treatment paradigms.

\section{Medical Treatment Strategies}

Medical treatment of epilepsy is required for surgical candidates to control seizures until surgical efficacy has been established. Drug treatment is also required in patients with tumor-related epilepsy, who are not candidates for surgical treatment. Treatment is often challenging, with up to $24 \%$ of patients requiring three or more antiepileptic drugs. $^{15}$

There are few randomized controlled trials comparing the efficacy of antiepileptic drugs in the treatment of this patient group. The choice of medication is therefore mainly determined by side-effect profile and drug interactions. As a general rule, antiepileptic drugs that induce cytochrome P450 hepatic enzymes should be avoided in patients who are likely to undergo chemotherapy (i.e., patients with highgrade glioma), as they can enhance the metabolism of chemotherapeutic agents, and they are often a contraindication to participating in clinical trials.

Interestingly, there is increasing evidence that some antiepileptic drugs may have direct antineoplastic actions that can improve survival in patients with high-grade glioma. Retrospective studies have shown that the use of valproate in patients with glioblastoma multiforme is associated with better survival, and that this effect depends upon treatment with temozolomide. ${ }^{16}$ Similarly, in vitro studies have shown that levetiracetam can suppress MGMT expression, with potential implications for the augmentation of temozolomide in adjuvant treatment of glioblastoma. ${ }^{17}$

The current first-line drugs in neuro-oncological practice are valproate and levetiracetam; however, many other antiepileptic drugs are also well tolerated, with few meaningful drug interactions, supporting their use as an adjunctive treatment in patients with tumor-related epilepsy.

\section{Surgical Treatment Strategies}

Long-term epilepsy-associated tumors rarely undergo malignant transformation; low-grade gliomas inevitably progress to higher grades over the course of the disease; high-grade gliomas are already malignant. In each case the surgical strategy should have clear aims, informed by the concept of an onco-epilepsy balance tailored to that individual patient (see - Fig. 3). In this section, we describe how best to approach these three groups of patients, striking the right onco-epilepsy balance. In each case, we assume a basic presurgical evaluation has taken place, and that seizure semiology, video telemetry, and neuropsychology are broadly concordant with the lesion in terms of likely seizure onset.

\section{Long-Term Epilepsy-Associated Tumors}

Although there are isolated reports in the literature of malignant transformation, LEATs generally behave in a very 


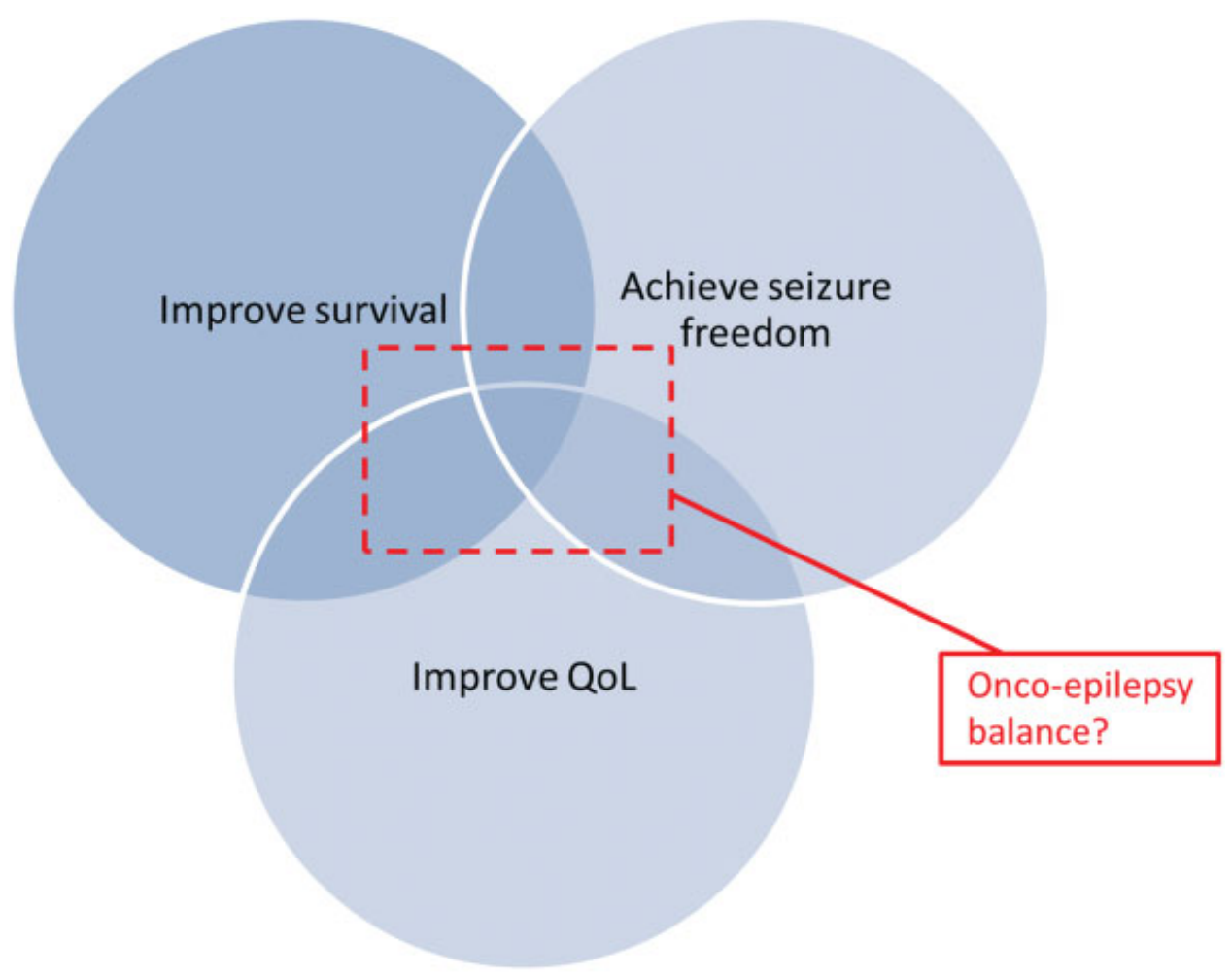

Fig. 3 Venn diagram to demonstrate the treatment aims and associated onco-epilepsy balance. QoL, quality of life.

benign fashion. ${ }^{18,19}$ Because these patients are likely to lead long lives, the priorities of surgical treatment should be to improve seizure control, but not at the expense of creating a neurologic deficit. The onco-epilepsy balance in this group should therefore be heavily biased in favor of curing the epilepsy. Here we deal with solitary LEATs first, followed by a discussion on LEATs in the context of dual pathology. = Fig. 4 gives an overview of the surgical treatment of LEATs.

\section{Solitary Long-Term Epilepsy-Associated Tumors}

Long-term epilepsy-associated tumors are often small, superficial, and well-circumscribed lesions, making them particularly amenable to total surgical resection. In theory, the pathophysiology of these tumors means that the epileptogenic zone should arise from within the tumor, and excellent results can be obtained in terms of seizure freedom following total lesionectomy. Interestingly, there is now good evidence that "solitary" glioneural tumors may in fact be associated with cryptogenic focal cortical dysplasia. ${ }^{20}$ Despite this, resections informed by invasive EEG (IC-EEG) do not seem to improve seizure freedom outcomes in this group. In most cases, the most pertinent question is therefore whether the lesion extends into eloquent cortex, and whether a total resection is feasible.

If the LEAT is small, solitary, and resides in noneloquent cortex, it is reasonable to proceed with a total lesionectomy. This can be done as an awake craniotomy with intraoperative functional mapping if there are any concerns regarding functional boundaries. There is no need in these cases to perform preresection IC-EEG to map out the likely epileptogenic zone because it likely resides within the tumor. There are considerable risks associated with IC-EEG, and there is no added benefit if the likely outcome will be a total lesionectomy. However, in the rare instances when the patient continues to have seizures following surgery, IC-EEG can then help to identify the epileptogenic zone in the peritumoral cortex. Further resection may ultimately demonstrate evidence of MRI-negative pathology, such as small residual tumor or focal cortical dysplasia.

If the LEAT resides or encroaches on eloquent cortex, the benefits of a total lesionectomy need to be balanced against the lifelong risk of neurologic deficit. In these cases we recommend pausing to consider whether surgical management is appropriate at all. If the patient wishes to continue along the surgical route, the next step is IC-EEG to map out the seizure-onset zone and irritative zone, and also to map the functional zone. ${ }^{21}$ Before implantation, these patients should undergo a thorough noninvasive presurgical evaluation, with data from advanced multimodality imaging used to inform the invasive monitoring. ${ }^{22}$ The aim of IC-EEG is to localize a part of the lesion where the seizures begin, and that can be safely resected, that is to inform a targeted subtotal lesionectomy. The relative strengths and weaknesses of subdural grid coverage versus stereo-EEG in general are discussed elsewhere. ${ }^{23}$ In these cases, tumors are often superficial, and mapping of the functional zone is limited to the surrounding cortex. We therefore recommend subdural grids for IC-EEG, because they provide better spatial resolution and make subsequent resection more straightforward to plan. In patients who progress to lesionectomy, there are clear 


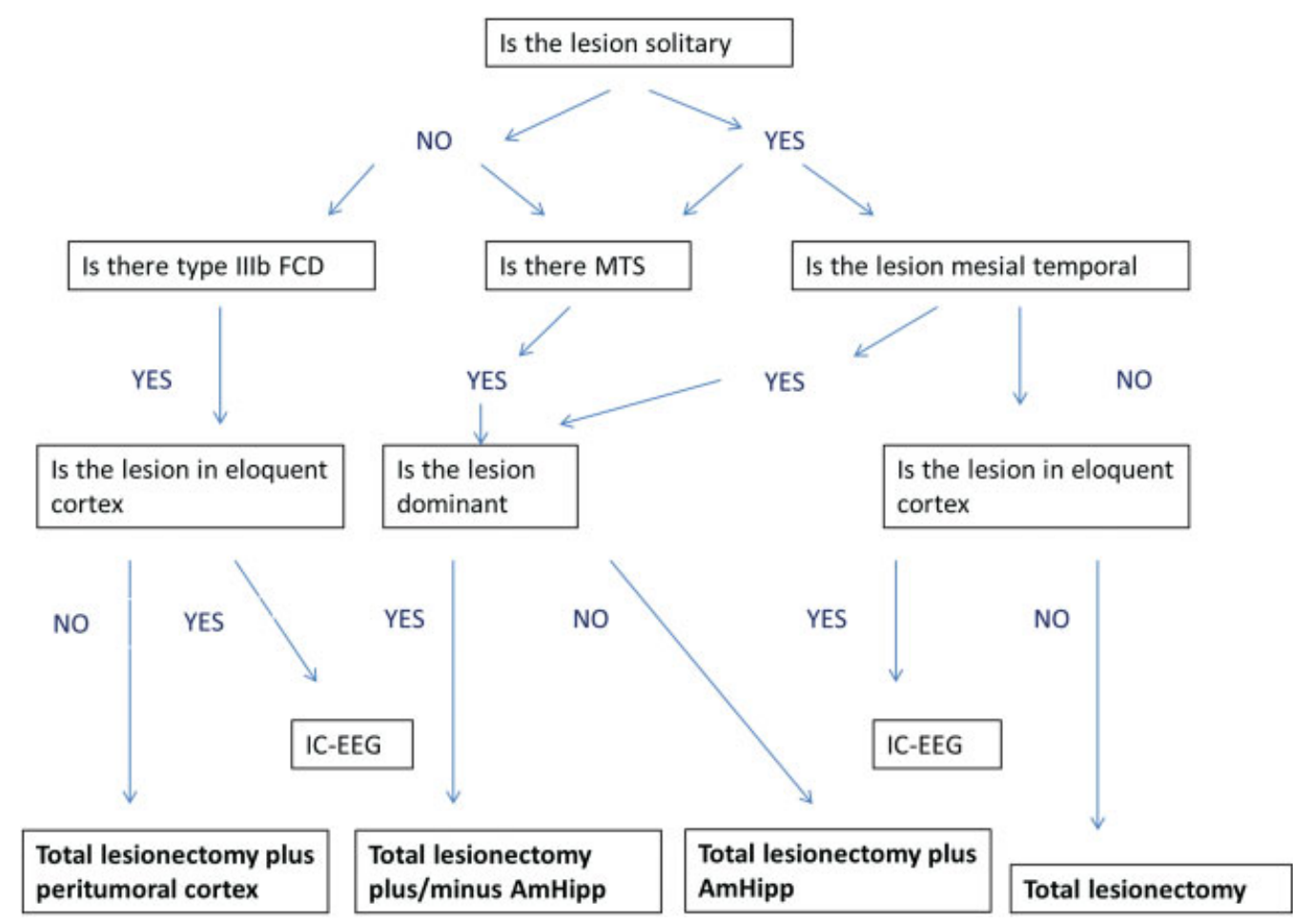

Fig. 4 An overview on the surgical treatment of long-term epilepsy associated tumors. AmHipp, amygdalohippocampectomy; EEG, electroencephalogram; FCD, focal cortical dysplasia; IC, intracranial; MTS, mesial temporal sclerosis.

advantages with awake craniotomy to confirm the extraoperative functional mapping.

\section{Long-Term Epilepsy Associated Tumors and Dual Pathology}

\section{Mesial Temporal Sclerosis}

Although LEATS can occur anywhere in the supratentorial compartment, they are most commonly found in the temporal lobe. There is a well-established link with mesial temporal LEATs and mesial temporal sclerosis, which is often not visible on the MRI. This presents a particular dilemma in surgical management; total lesionectomy coupled with removal of the mesial temporal structures (amygdala and hippocampus) gives superior results for seizure freedom over just lesionectomy, ${ }^{9}$ but is associated with higher risks of postoperative neuropsychological deficits in visual and verbal memory.

As stated previously, each patient should undergo rigorous presurgical evaluation, including neuropsychological testing to predict physiological reserve and the likely impact on quality of life, and surgical management should be tailored to individual needs. In general, if the lesion is on the nondominant side, the cognitive risk associated with an amygdalohippocampectomy is considerably less, favoring the removal of both the lesion and the mesial temporal structures, with high chances of achieving seizure freedom. However, if the lesion is on the dominant side and the patient has high physiological reserve, the cognitive risk associated with amygdalohippocampectomy is greater, and can significantly affect the patients' quality of life. In these instances, the pragmatic approach is a total lesionectomy, preserving the mesial structures if they appear structurally and functionally normal. It should be discussed preoperatively with these patients that this approach carries a slightly lower chance of achieving seizure freedom. However if the patients are not seizure-free following surgery, further surgical options remain open. This may be extension of the resection to include the mesial structures if implicated, or IC-EEG to determine the epileptogenic zone in surrounding cortex.

\section{Focal Cortical Dysplasia}

The ILAE classification of focal cortical dysplasia includes type IIIb, where cortical lamination abnormalities lie concurrent to a tumor. ${ }^{24}$ Most commonly this occurs with LEATs, although they can also occur with low-grade gliomas.

The treatment challenge in these cases is to determine whether the epileptogenic zone arises from the tumor or the peri-tumoral dysplasia. For small tumors in non-eloquent areas with limited focal cortical dysplasia, the precise identification of the epileptogenic zone is probably unnecessary, and the pragmatic approach is to perform a lesionectomy and extended resection of the peri-tumoral cortex to include the adjacent dysplasia. For larger tumors with encroachment into eloquent cortex, this extended resection is not possible and precise determination of the epileptogenic zone is crucial. In these instances, IC-EEG is the first step to determine likely epileptogenic and functional zones. Further surgical treatment can then be tailored to that individual according to the findings of the invasive study. 


\section{Low-Grade Glioma}

Malignant transformation in low-grade glioma is certain, with a median survival of 8.2 years. ${ }^{3}$ Although the prognosis of these tumors remains poor, there have been some advances in our understanding that inform modern surgical management.

Historically, it was thought that the natural history of these tumors was poor irrespective of the surgical treatment. However, it is becoming increasingly accepted that the extent of resection is a major predictor of length of survival, with successively greater improvements associated with extent of resection over $70 \%{ }^{11}$ A further change to our understanding is in what constitutes a total resection. The convention has been to describe this radiologically as the volume defined by gadolinium enhancement on the T1-weighted MRI. However, it is well established that tumor cells infiltrate white matter beyond these margins, as evidenced by the large signal change on T2 fluid-attenuated inversion recovery (FLAIR)
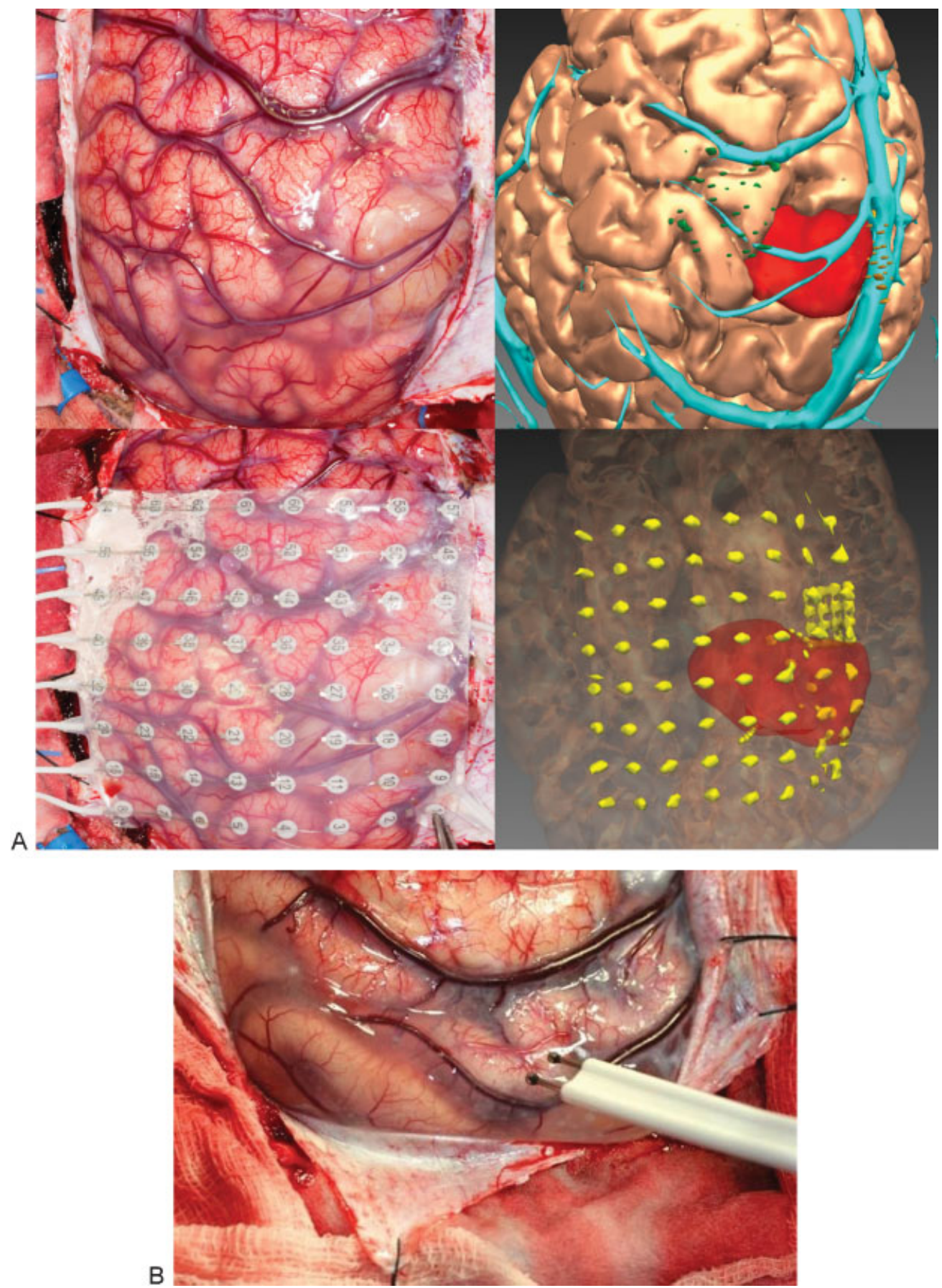

Fig. 5 (A) Intraoperative photograph of subdural grid over lesion, a dysembryoplastic neuroepithelial tumor (DNET), for extraoperative mapping; top left: photograph of left frontoparietal cortex with DNET straddling motor cortex, top right: three-dimensional (3D) computer model of cortex (pink), lesion (red), cortical veins (cyan), hand transcranial magnetic stimulation (green), and leg transcranial magnetic stimulation (orange); bottom left: intraoperative photograph of left frontoparietal cortex with overlying subdural grid; bottom right: 3D computer model of cortex, lesion, implanted electrode contacts (yellow). (B) Photograph of intraoperative mapping by cortical stimulation in an awake patient. 
MRI. The onus in these patients is therefore shifting away from biopsy toward total resection, and even supratotal resection, where the resection is only limited by functional boundaries. ${ }^{25}$ Thus, the onco-epilepsy balance in this group has shifted toward maximal oncological clearance, with the additional benefit of large-volume histological samples for accurate grading and genetic profiling.

Total and supratotal resections depend on the identification and preservation of functional boundaries. This can be achieved in two ways. One way is to use IC-EEG to perform extraoperative mapping of eloquent cortex. Subdural grids are best suited to this because they generate good spatial coverage, and their placement can be informed by functional MRI and gyral anatomy on 3-dimensional computer models. ${ }^{22}$ The advantage of this technique is that the mapping can be done under controlled conditions, with the patient fully conscious. This is often employed in the determination of the functional zone in children (- Fig. 5A).

However, there are several disadvantages to this technique. Usually, subdural grids have $1-\mathrm{cm}$ spacing between contacts, making for limited accuracy. The implantation of a subdural grid carries substantial risks of infection and hemorrhage, and commits the patient to undergoing two surgical procedures. Perhaps most importantly, extraoperative mapping only samples cortex and provides no information on the underlying white matter tracts. This is crucial; White matter connectivity is the route that the pathology migrates along, and also represents the limitation of brain plasticity potential.

The alternative mapping method is intraoperative mapping, eliciting responses in the awake patient during surgery using cortical and subcortical stimulation. The key advantage with this technique is that it allows for the functional mapping of the underlying white matter tracts. A recent meta-analysis, based on 20 years of glioma surgery and over 8,000 cases, has shown that the use of intraoperative mapping significantly increases the extent of resection and also reduces the rate of postoperative neurologic deficits in eloquent areas. ${ }^{26}$ It is possible to map complex functions such as language, spatial cognition, calculation, judgment, and executive functions with this technique. This requires an integrated approach, with specialist testing, the correct organizational framework, and excellent anesthesia (- Fig. 5B). ${ }^{27}$

\section{High-Grade Glioma}

In patients with high-grade glioma, seizure control is rarely the key issue. Treatment aims for these patients is to prolong survival and maintain quality of life. The optimum treatment is surgical resection followed by adjuvant chemoradiotherapy. However, seizures are obviously associated with a poor quality of life, and the potential to improve seizure control should be factored into the decision to perform a radical tumor resection.

\section{Summary}

In summary, it is simplistic to assume that tumor-related epilepsy should always be managed by lesionectomy. The treatment depends on tumor type and associated pathophysiology, and should be tailored to individual patients, based on discussions around feasible aims. The oncoepilepsy balance distinguishes LEATs from low-grade gliomas. The emphasis with LEATs is on epilepsy control; this group has an excellent prognosis, with the onus on early diagnosis and surgical management to achieve improved rates of seizure freedom. The treatment of LEATs is complicated by the possibility of dual pathology. By contrast, the emphasis with low-grade gliomas is on oncological clearance; this group has a worse prognosis, but also relies on early diagnosis coupled with supratotal resection to achieve improved survival rates. In both groups, functional preservation is critical. The use of IC-EEG to inform resection should be rationed to particular cases, where there is a clear practical benefit that will inform any subsequent resection. Medical management of tumor-related epilepsy is an important adjunct to surgical management, with the choice of antiepileptic drugs largely determined by side-effect profile and drug interactions. Care of these patients is complex, and requires a multidisciplinary team approach.

\section{Acknowledgments}

Mark Nowell is supported by the Department of Health and Wellcome Trust through the Health Innovation Challenge Fund (HICF-T4-275, Program Grant 97914) and is working in collaboration with Medtronic, Inc., on a project entitled Novel Multimodality Imaging in Epilepsy Surgery. We are grateful to the Wolfson Trust and the Epilepsy Society for supporting the Epilepsy Society MRI scanner. This work was supported by the National Institute for Health Research (NIHR) University College London Hospitals Biomedical Research Centre (BRC). Andrew McEvoy has received support from UCB, Baxter, and Cyberonics.

\section{References}

1 Japp A, Gielen GH, Becker AJ. Recent aspects of classification and epidemiology of epilepsy-associated tumors. Epilepsia 2013;54 (Suppl 9):5-11

2 Cowie CJ, Cunningham MO. Peritumoral epilepsy: relating form and function for surgical success. Epilepsy Behav 2014;38:53-61

3 Citow JS, Macdonald RL, Refai D. Comprehensive Neurosurgery Board Review. 2nd ed. New York, Stuttgart: Thieme; 2009

4 Luyken C, Blümcke I, Fimmers R, et al. The spectrum of longterm epilepsy-associated tumors: long-term seizure and tumor outcome and neurosurgical aspects. Epilepsia 2003;44(6): $822-830$

5 Stupp R, Mason WP, van den Bent MJ, et al; European Organisation for Research and Treatment of Cancer Brain Tumor and Radiotherapy Groups; National Cancer Institute of Canada Clinical Trials Group. Radiotherapy plus concomitant and adjuvant temozolomide for glioblastoma. N Engl J Med 2005;352(10):987-996

6 Cossu M, Fuschillo D, Bramerio M, et al. Epilepsy surgery of focal cortical dysplasia-associated tumors. Epilepsia 2013;54 (Suppl 9):115-122

7 Pallud J, Capelle L, Huberfeld G. Tumoral epileptogenicity: how does it happen? Epilepsia 2013;54(Suppl 9):30-34

8 Guerrini R, Rosati A, Giordano F, Genitori L, Barba C. The medical and surgical treatment of tumoral seizures: current and future perspectives. Epilepsia 2013;54(Suppl 9):84-90 
9 Englot DJ, Berger MS, Barbaro NM, Chang EF. Factors associated with seizure freedom in the surgical resection of glioneuronal tumors. Epilepsia 2012;53(1):51-57

10 Englot DJ, Berger MS, Barbaro NM, Chang EF. Predictors of seizure freedom after resection of supratentorial low-grade gliomas. A review. J Neurosurg 2011;115(2):240-244

11 Ius T, Isola M, Budai R, et al. Low-grade glioma surgery in eloquent areas: volumetric analysis of extent of resection and its impact on overall survival. A single-institution experience in 190 patients: clinical article. J Neurosurg 2012;117(6):1039-1052

12 Sanai N, Chang S, Berger MS. Low-grade gliomas in adults. J Neurosurg 2011;115(5):948-965

13 Kim JW, Park CK, Park SH, et al. Relationship between radiological characteristics and combined $1 \mathrm{p}$ and $19 q$ deletion in World Health Organization grade III oligodendroglial tumours. J Neurol Neurosurg Psychiatry 2011;82(2):224-227

14 Schiff D. Low-grade gliomas. J Neurosurg 2011;115(5):945-947, author reply 947

15 van Breemen MS, Rijsman RM, Taphoorn MJ, Walchenbach R, Zwinkels H, Vecht CJ. Efficacy of anti-epileptic drugs in patients with gliomas and seizures. J Neurol 2009;256(9):1519-1526

16 Weller M, Gorlia T, Cairncross JG, et al. Prolonged survival with valproic acid use in the EORTC/NCIC temozolomide trial for glioblastoma. Neurology 2011;77(12):1156-1164

17 Bobustuc GC, Baker CH, Limaye A, et al. Levetiracetam enhances p53-mediated MGMT inhibition and sensitizes glioblastoma cells to temozolomide. Neuro-oncol 2010;12(9):917-927

18 Bendersky D, Tedesco N, Christiansen S, García MdelC, Ciraolo C. Epilepsy due to a temporal ganglioglioma and its subsequent malignant transformation into a primitive neuroectodermal tumor. Surg Neurol Int 2012;3:79
19 Moazzam AA, Wagle N, Shiroishi MS. Malignant transformation of DNETs: a case report and literature review. Neuroreport 2014; 25(12):894-899

20 Rosenow F, Menzler K. Invasive EEG studies in tumor-related epilepsy: when are they indicated and with what kind of electrodes? Epilepsia 2013;54(Suppl 9):61-65

21 Rosenow F, Lüders H. Presurgical evaluation of epilepsy. Brain 2001;124(Pt 9):1683-1700

22 Rodionov R, Vollmar C, Nowell M, et al. Feasibility of multimodal 3D neuroimaging to guide implantation of intracranial EEG electrodes. Epilepsy Res 2013;107(1-2):91-100

23 Sweet JA, Hdeib AM, Sloan A, Miller JP. Depths and grids in brain tumors: implantation strategies, techniques, and complications. Epilepsia 2013;54(Suppl 9):66-71

24 Santos MV, de Oliveira RS, Machado HR. Approach to cortical dysplasia associated with glial and glioneuronal tumors (FCD type IIIb). Childs Nerv Syst 2014;30(11):1869-1874

25 Duffau $H$. The challenge to remove diffuse low-grade gliomas while preserving brain functions. Acta Neurochir (Wien) 2012; 154(4):569-574

26 De Witt Hamer PC, Robles SG, Zwinderman AH, Duffau H, Berger MS. Impact of intraoperative stimulation brain mapping on glioma surgery outcome: a meta-analysis. J Clin Oncol 2012;30(20): 2559-2565

27 Duffau H. Brain mapping in tumors: intraoperative or extraoperative? Epilepsia 2013;54(Suppl 9):79-83

28 Thom M, Toma A, An S, et al. One hundred and one dysembryoplastic neuroepithelial tumors: an adult epilepsy series with immunohistochemical, molecular genetic, and clinical correlations and a review of the literature. J Neuropathol Exp Neurol 2011;70(10):859-878 